\title{
Cation-Responsive and Photocleavable Hydrogels from Noncanonical Amphiphilic DNA Nanostructures
}

\author{
Giacomo Fabrini, Aisling Minard, Ryan A. Brady, Marco Di Antonio,* and Lorenzo Di Michele*
}

Cite This: Nano Lett. 2022, 22, 602-611

Read Online

\section{ACCESS | Lill Metrics \& More | 回 Article Recommendations | si Supporting Information}

ABSTRACT: Thanks to its biocompatibility, versatility, and programmable interactions, DNA has been proposed as a building block for functional, stimuli-responsive frameworks with applications in biosensing, tissue engineering, and drug delivery. Of particular importance for in vivo applications is the possibility of making such nanomaterials responsive to physiological stimuli. Here, we demonstrate how combining noncanonical DNA G-quadruplex (G4) structures with amphiphilic DNA constructs yields nanostructures, which we termed "Quad-Stars", capable of assembling into responsive hydrogel particles via a straightforward, enzyme-free, onepot reaction. The embedded G4 structures allow one to trigger and control the assembly/disassembly in a reversible fashion by adding or removing $\mathrm{K}^{+}$ions. Furthermore, the hydrogel aggregates can be photo-disassembled upon near-UV irradiation in the presence of a porphyrin photosensitizer. The combined reversibility of assembly, responsiveness, and cargo-loading capabilities of the hydrophobic moieties make Quad-Stars a promising candidate for biosensors and responsive drug delivery carriers.

KEYWORDS: Amphiphilic DNA, stimuli-responsive hydrogels, G-quadruplexes, self-assembly, DNA nanotechnology

$\mathrm{N}$ ucleic acids have proven to be valuable building materials for the self-assembly of $2 \mathrm{D}$ and 3D architectures, nanomachines, and functional nanomaterials. ${ }^{1-3}$ This success is not surprising, as nucleic acids benefit from superior biocompatibility, programmable interactions, and a decreasing production cost.

DNA-based hydrogels, in particular, have shown promise in applications as varied as biosensing, ${ }^{6,7}$ drug delivery, ${ }^{8-10}$ synthetic biology, ${ }^{11}$ and artificial cells. ${ }^{12}$ Key characteristics sought in DNA-based hydrogels include fast, scalable, and costeffective production, and stimuli-responsiveness, particularly in the form of triggered assembly/disassembly.

Hybrid DNA-polymer hydrogels, usually constructed from poly(acrylamide) (PAAm) or poly(ethylene glycol) (PEG) cross-linked via DNA side chains, have proven highly successful as biosensors and carriers, as they can respond to a wide range of stimuli, including thermal denaturation, ${ }^{6}$ strand displacement, ${ }^{13}$ small molecules opening DNA-aptamer bridges, ${ }^{14}$ and metal ions triggering cleavage through DNAzymes. ${ }^{15}$ Similar hydrogels have been engineered to undergo location-addressable swelling via reconfigurable DNA cross-links, applicable in soft robotics. ${ }^{16}$ Replacing DNA duplexes with noncanonical i-motifs ${ }^{17}$ and G-quadruplexes $(\mathrm{G} 4 \mathrm{~s})^{18-20}$ further enables responsiveness to $\mathrm{pH}$ and cation concentration, respectively. A combination of double-stranded DNA (dsDNA) linkers and i-motifs has been used to produce shape-memory DNA hydrogels, with potential applications in tissue scaffolding and engineering. ${ }^{21}$ However, the hybrid approach suffers from potentially toxic breakdown products, particularly in the case of acrylamide-based hydrogels, ${ }^{22}$ and it requires an additional production step to covalently link DNA and polymer.

Conversely, all-DNA hydrogels could feature higher biocompatibility and biodegradability, ${ }^{23}$ while simplifying the production route, and have been applied to controlled drug release $^{24}$ and cell-free protein production. ${ }^{25,26}$ The inclusion of i-motifs ${ }^{27}$ and triplexes ${ }^{28}$ enables $\mathrm{pH}$-triggered, reversible sol/ gel transitions, ${ }^{29}$ while DNA-aptamer bridges allow for disassembly upon binding small molecule targets. ${ }^{30,31}$ Currently, though, $\mathrm{pH}$-triggered assembly/disassembly is the only example of a reversible structural response driven in allDNA hydrogels by a physiologically relevant stimulus, limiting biomedical applicability.

Recently, we introduced a novel class of amphiphilic DNA nanostructures, dubbed C-Stars, ${ }^{32-34}$ that combine the programmability of DNA with the robust self-assembly of amphiphiles. C-Stars consist of branched DNA junctions (nanostars) featuring a cholesterol moiety at the end of each

Received: August 26, 2021

Revised: December 1, 2021

Published: January 13, 2022

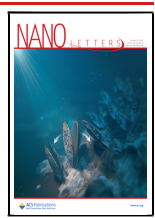




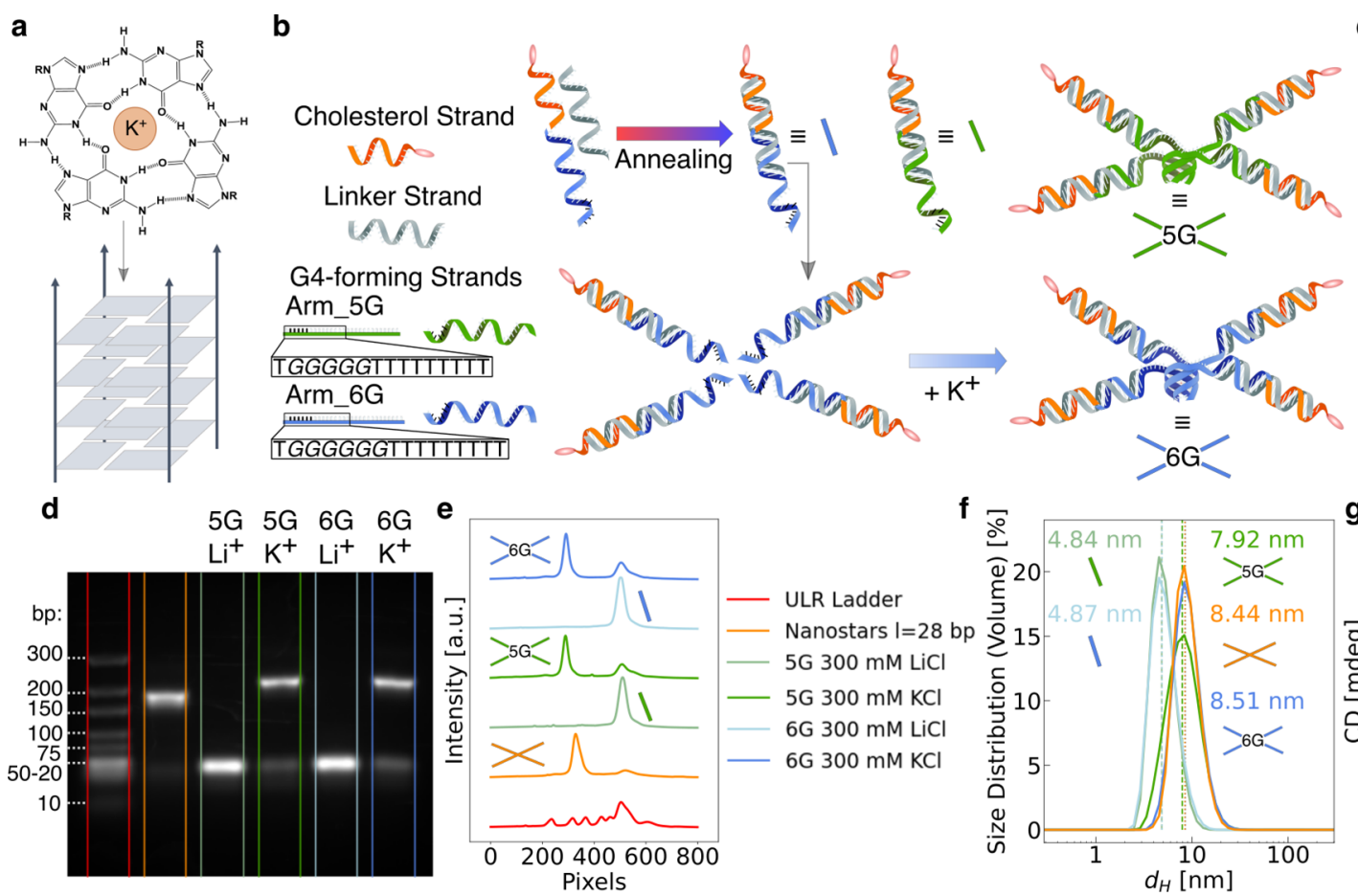

C

d
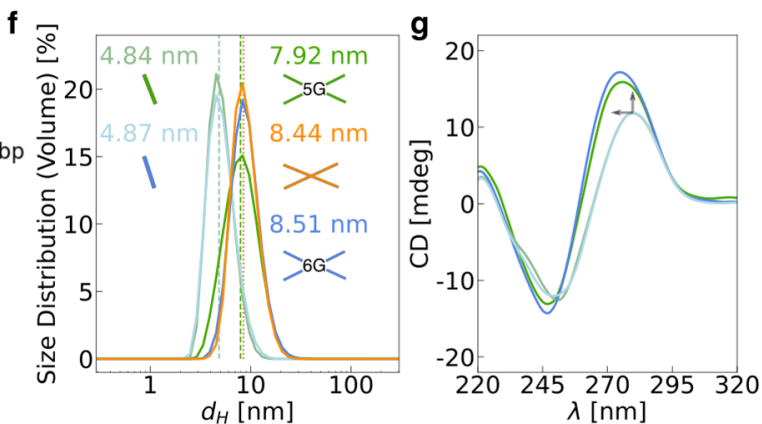

Figure 1. Design and characterization of amphiphilic DNA Quad-Stars. (a) Schematic of a G-quartet (top) and a parallel tetramolecular G4 (bottom). (b) Quad-Stars assemble from four duplex arms, each made of three strands. During the annealing process, single-stranded DNA components hybridize into duplex arms, but G4 formation and assembly of the designed Quad-Star occurs only in the presence of $\mathrm{K}^{+}$ions. Two design variations, labeled as $5 \mathrm{G}$ and $6 \mathrm{G}$ ( 5 or 6 guanines in the G-run of the G4 forming sequence), are investigated. (c) Schematic of C-Stars ${ }^{32}$ with arm length $l=28$ base pairs (same as Quad-Stars). C-Stars and Quad-Stars share the same cholesterol-functionalized strands. (d) Agarose gel electrophoresis confirms that formation of individual, noncholesterolized Quad-Stars is $\mathrm{K}^{+}$-dependent. Slower bands for both $5 \mathrm{G}$ (green) and 6G (blue) only appear when motifs are annealed in $\mathrm{KCl}(300 \mathrm{mM})$, and their mobility is comparable to that of four-arm nanostars with an arm length of $28 \mathrm{bp}$ (orange). (e) Intensity profiles corresponding to the gel in panel d. (f) Volume-based size distribution obtained via dynamic light scattering (DLS) further substantiating the formation of Quad-Stars in $\mathrm{KCl}$, with a net shift from the size distribution in LiCl. As expected from panel $\mathrm{d}$, the size of assembled motifs in $\mathrm{KCl}$ is comparable that of four-arm nanostars in $300 \mathrm{mM} \mathrm{NaCl}$. (g) Circular dichroism (CD) proving that self-assembly relies on the formation of tetramolecular parallel G4s. Overlaid CD spectra in $\mathrm{LiCl}$ (faint colors) and $\mathrm{KCl}$ (saturated colors) clearly display the $\mathrm{K}^{+}$-induced shift (highlighted via arrows) in maximum and minimum toward $\approx 263$ and $240 \mathrm{~nm}$, respectively, as typical of parallel G4 structures. Samples for all reported measurements were prepared at [oligonucleotide] $=20 \mu \mathrm{M}$.

arm, which enables the formation of crystalline frameworks or amorphous hydrogels sustained by hydrophobic interactions. DNA-based frameworks have been proposed as a general strategy to program designer nanomaterials, such as supramolecular assemblies of nanoparticles, ${ }^{35,36}$ quantum dots, ${ }^{37,38}$ and proteins. ${ }^{39}$ Compared to these approaches, which are fully reliant on base-pairing or stacking interactions, the hydrophobicity-mediated self-assembly principle of C-Stars warrants enhanced robustness against design variations and changes in environmental conditions, making it possible to tune porosity, capture/release proteins, and trigger disassembly through toehold-mediated strand displacement. The previously demonstrated disassembly response, however, requires nonbiologically available single-stranded DNA as input and is thus not directly applicable to in vivo scenarios.

Here, we expand upon the responsiveness of amphiphilic DNA nanostars by combining their robustness and versatility with a cation-dependent DNA G4 motif. The resulting nanostructures, which we term Quad-Stars, form nanoporous hydrogels responsive to biologically relevant $\mathrm{K}^{+}$cations. The materials assemble in a simple, enzyme-free, one-pot reaction when exposed to $\mathrm{K}^{+}$ions, the chelation of which leads to disassembly. The amphiphilicity of the networks allows for loading of small hydrophobic molecules, which can then be released upon disassembly. Besides $\mathrm{K}^{+}$chelation, the material can also be disassembled upon addition of a G4-binding porphyrin photosensitizer and exposure to long-wave UV light, the combination of which disrupts the quadruplex through guanine photo-oxidation. ${ }^{40-44}$ This additional mechanism is irreversible but enables precise spatial control over the response. Owing to the combined assembly reversibility, multistimuli-responsiveness, cargo-loading capabilities, and stability in model physiological conditions, we believe that Quad-Star hydrogels hold significant potential for applications such as smart biosensors and drug delivery.

Quad-Stars comprise four identical duplex “arms” bearing a cholesterol end-modification which, under suitable conditions, can join via a tetramolecular parallel G4 motif, as shown in Figure 1a,b. G-quadruplexes are noncanonical secondary structures that can form in guanine-rich nucleic acid strands unimolecularly, bimolecularly, or, as in Quad-Stars, tetramolecularly, i.e., between four distinct strands. ${ }^{45}$ Depending on the strand orientation, G4s can be parallel, antiparallel, or hybrid. They comprise a series of stacked G-quartets interacting via Hoogsteen hydrogen bonding, as depicted in Figure 1a (top). Alkali metal ions, particularly $\mathrm{K}^{+}$, coordinate guanines, templating and stabilizing G-quartets and their stacking into G4s. This grants G4s, and in turn Quad-Stars, a degree of responsiveness with respect to cationic environments. 
As shown in Figure 1b, each Quad-Star arm assembles from three DNA strands, one bearing a cholesterol moiety, connected via a triethylene glycol (TEG) spacer, another acting as linker, and a third terminating with a 5 or 6 nucleotide (nt)-long guanine run flanked by thymines ( $\mathrm{T}$ GGGGG $\mathrm{T}$ or $\mathrm{T}$ GGGGGG $\mathrm{T}$; see zoomed inset in Figure $1 b)$, a sequence capable of assembling into tetramolecular parallel G4s under physiological conditions. ${ }^{46}$ In the remainder of the text we refer to these designs as $5 \mathrm{G}$ and $6 \mathrm{G}$, respectively.

In the assembled arm, a poly- $T$ spacer is present between the G-run and the duplex portion to increase flexibility at the G4 site. Thymine-spacer lengths of 3,5, 7, and 9 nucleotides were tested, and minimal differences were observed in the QuadStar self-assembly behavior. In the remainder of the main text we report results for 9T spacers, while analogous findings for different lengths are featured in the SI.

Assembled Quad-Stars are topologically analogous to previously introduced C-Stars (Figure 1c), with the key difference being that Quad-Star arms are held together by a stimuli-responsive G4 motif rather than an irreversible fourway junction. Similarly to C-Stars, ${ }^{32,33}$ we therefore expect Quad-Stars to self-assemble into nanoporous networks owing to the clustering of cholesterol moieties into micelle-like structures, coordinated and linked by the tetravalent DNA motifs. Unlike C-Stars, however, we expect Quad-Stars and Quad-Star networks to respond to changes in ionic environment, self-assembling at sufficiently high concentration of G4promoting $\mathrm{K}^{+}$ions (and $\mathrm{Na}^{+}$to a lower extent), while being destabilized by cations with a lower affinity for the G4 microenvironment, such as $\mathrm{Li}^{+}$or $\mathrm{Mg}^{2+}$ (Figure $1 \mathrm{~b}$ ).

To verify the self-assembly of Quad-Stars into the sought branched architectures at thermodynamic equilibrium and under suitable ionic conditions, samples of both the $5 \mathrm{G}$ and 6G designs were slowly annealed in TE buffer containing 300 $\mathrm{mM} \mathrm{LiCl} / \mathrm{NaCl} / \mathrm{KCl}$ or $2 \mathrm{mM} \mathrm{MgCl}_{2}$ (see Methods, SI). Network formation was here prevented by replacing cholesterol-tagged strands with nonfunctionalized ones having identical sequence.

For both 5G and 6G samples annealed in $\mathrm{LiCl}$, agarose gel electrophoresis (AGE) shows a single band corresponding to disjoined nanostar arms. Samples annealed in $\mathrm{KCl}$, instead, feature a second, slower band with mobility comparable to that of four-arm junctions (nanostars) with $28 \mathrm{bp}$ arm length, which can thus be ascribed to correctly formed Quad-Stars (Figure 1d,e). Correct assembly is confirmed by dynamic light scattering (DLS) (Figure 1f), which demonstrates a cationdependent size shift for both $5 \mathrm{G}$ and 6G designs, with samples in $\mathrm{KCl}$ showing the same average hydrodynamic diameter as four-arm nanostars with the same arm length. As shown by AGE and DLS, samples annealed in $2 \mathrm{mM} \mathrm{MgCl}_{2}$ (Figures $\mathrm{S} 1$ and S2, SI) and $300 \mathrm{mM} \mathrm{NaCl}$ (Figures S3 and S4, SI) exhibit a lower degree of Quad-Star assembly for the 6G design and no assembly for $5 \mathrm{G}$, hence pointing at a degree of quadruplex stabilization intermediate between $\mathrm{Li}^{+}$and $\mathrm{K}^{+}$, in agreement with literature. ${ }^{47}$ When reducing the concentration of monovalent cations to $100 \mathrm{mM}$, assembly was only observed for $6 \mathrm{G}$ designs in $\mathrm{K}^{+}$-rich buffers (Figures S3 and S4, SI).

Circular dichroism (CD) spectra were used to confirm the presence, and determine the topology, of the G4s (Figure 1g). Compared to those prepared in $\mathrm{LiCl}$, samples annealed in $\mathrm{KCl}$ present a clear shift in peak and valley positions toward 263 and $240 \mathrm{~nm}$, respectively, accompanied by an amplitude increase, indicating the formation of parallel G4s, as intended.
Results for 5G and 6G Quad-Stars with different poly-T spacer lengths are collated in Figures S5-S7 (SI). Quad-Star designs with shorter G-runs (4G) were also tested and found incapable of assembling even in $\mathrm{KCl}$ buffers, producing results similar to those of negative controls where guanines are replaced by thymines (see Figures S5-S7, SI).

To explore the ability of Quad-Stars to isothermally respond to changes in ionic conditions, noncholesterolized Quad-Stars were annealed in $300 \mathrm{mM} \mathrm{LiCl}$ buffers, hence resulting in dispersed duplex arms, before triggering assembly via rapid injection of $\mathrm{KCl}$ (see Methods, SI). The assembly transient was then tracked by time-resolved fluorescence using $N$-methyl mesoporphyrin IX (NMM), whose quantum yield dramatically increases upon preferential complexation with parallel G4s. ${ }^{48-50}$

The obtained fluorescence intensity profiles monotonically increase after $\mathrm{KCl}$ injection, demonstrating $\mathrm{G} 4$ formation and isothermal assembly of Quad-Stars, and could be fitted to a simple model ${ }^{46}$ (see Methods, SI) to extract the assembly halflife, $t_{1 / 2}$, as exemplified in Figure $2 \mathrm{a}$. The values obtained at the
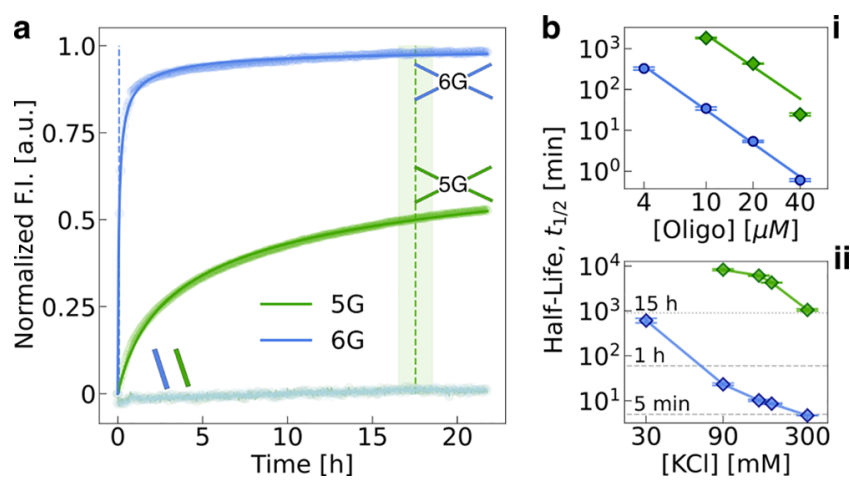

Figure 2. Kinetics of G4-dependent formation in soluble Quad-Stars via NMM fluorimetry. (a) Kinetic profiles of G4-folding at [Oligo] = $20 \mu \mathrm{M}$ (Oligo = oligonucleotide) and $[\mathrm{KCl}]=300 \mathrm{mM}$. As G4s form, NMM stacks on G-quartets and undergoes fluorescence emission enhancement. Normalized fluorimetry data are presented as mean (data points, one marker every three points shown) \pm standard deviation (shaded region) and fits (solid lines) to theoretical tetramolecular kinetics. ${ }^{46}$ Half-life values are reported as mean (vertical lines) \pm standard deviation (shaded intervals). Fainter colored profiles at the bottom are negative controls (no $\mathrm{KCl}$ injection), reported as average data points only. (b) Half-life of formation Vs (i) [Oligo] at fixed $[\mathrm{KCl}]=300 \mathrm{mM}$ and (ii) $[\mathrm{KCl}]$ at fixed $[$ Oligo $]=20 \mu \mathrm{M}$. (i) Data have been linearly fitted (solid lines) in $\log$ - $\log$ scale according to power law $t_{1 / 2}=A[\text { Oligo }]^{1-n}$ to extract the reaction order from the fitting slope, resulting in $n=3.605 \pm$ 0.740 for $5 \mathrm{G}\left(R^{2}=0.911\right)$ and $n=3.711 \pm 0.127\left(R^{2}=0.996\right)$ for $6 \mathrm{G}$. Both values closely match the expected stoichiometric value of 4 . Note that, owing to the prohibitively slow formation rate, assembly of the 5G motifs could not be tested at the lowest oligonucleotide and $\mathrm{KCl}$ concentrations used for 6G. Data in panel $\mathrm{b}$ are presented as mean (data points) \pm standard deviation (error bars). Negative controls in panel a are averages of independent duplicates. All other data have been averaged across (at least) triplicates. See Figure S8 (SI) for kinetic profiles for panel $b$.

target conditions of $20 \mu \mathrm{M}$ oligonucleotide concentration ([Quad-Star] $=5 \mu \mathrm{M}$ ) and $300 \mathrm{mM} \mathrm{KCl}$ clearly indicate that longer G-runs translate into faster assembly, with $t_{1 / 2} \sim 5 \mathrm{~min}$ for $6 \mathrm{G}$ and $t_{1 / 2} \sim 15 \mathrm{~h}$ for $5 \mathrm{G}$. This trend is consistent with previous literature reports ${ }^{46,51,52}$ and can be rationalized as the result of a nucleation-zipping assembly pathway. ${ }^{53,54}$ Within 
a

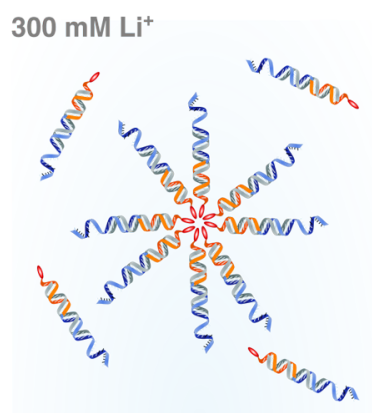

$2 \mathrm{mM} \mathrm{Mg}^{2}$

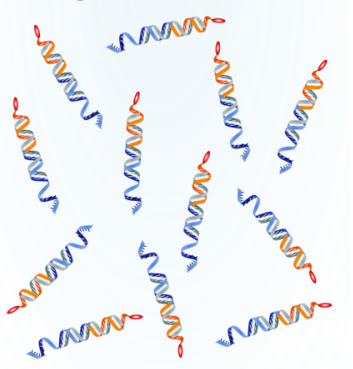

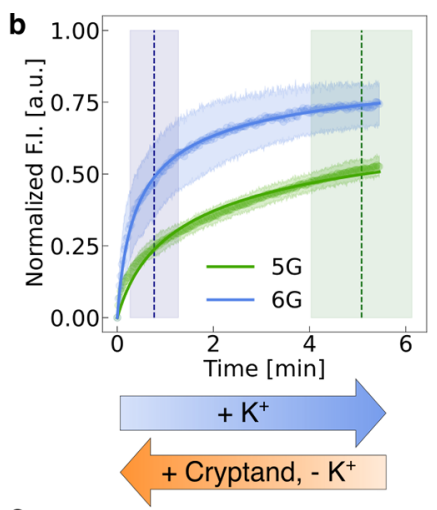

C

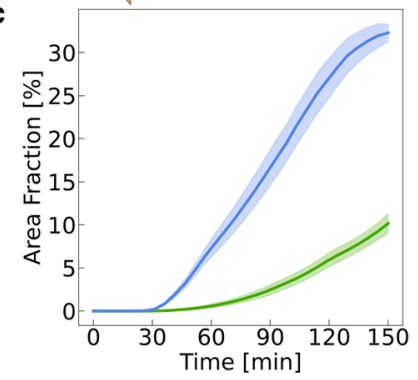

d

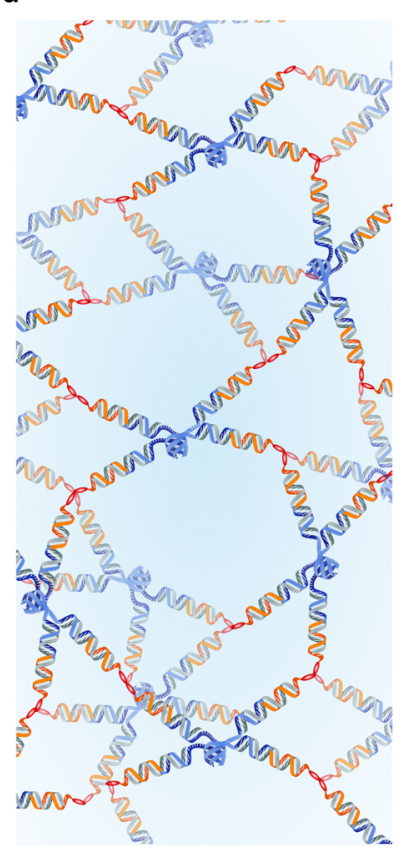

e

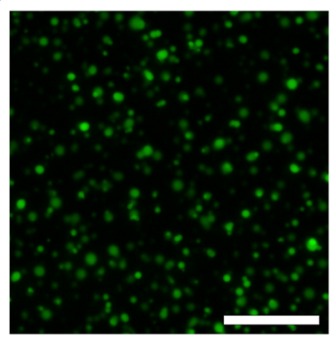

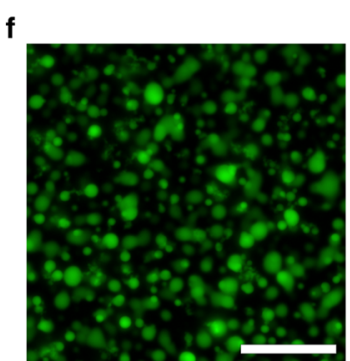

Cycle 3
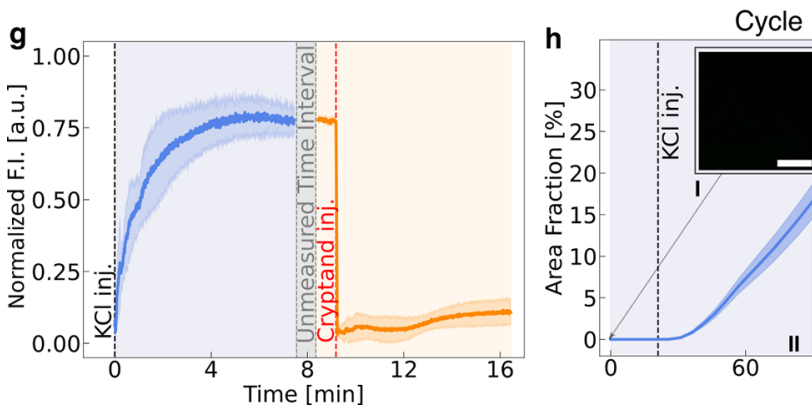

Cycle 1
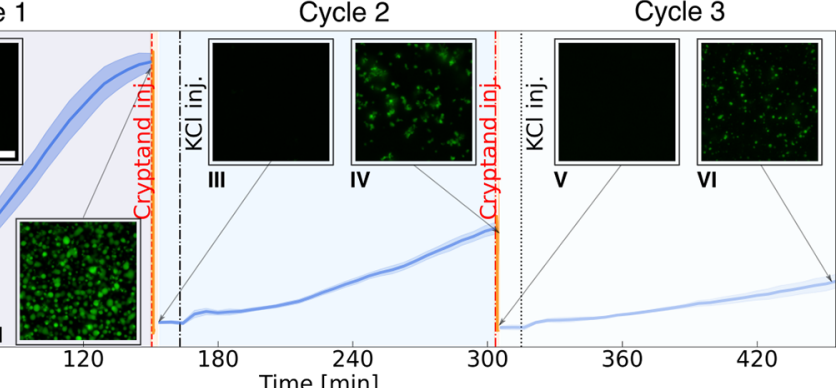

Figure 3. Reversible, isothermal assembly of Quad-Stars via NMM fluorimetry and epifluorescence microscopy. (a) Schematic representation of cholesterol-induced micelles and individual double-stranded DNA arms formed after annealing in $300 \mathrm{mM} \mathrm{LiCl}$ and $2 \mathrm{mM} \mathrm{MgCl}_{2}$, respectively (see Figure S9, SI). Presented data refer to samples annealed in $2 \mathrm{mM} \mathrm{MgCl}_{2}$. (b) Kinetic profiles of G4-folding in cholesterolized Quad-Stars via NMM fluorimetry. Normalized fluorimetry data are presented as mean (data points, one marker every three points shown) \pm standard deviation (shaded region) and fits (solid lines) to theoretical tetramolecular kinetics. ${ }^{46}$ Half-life values are reported as mean (vertical lines) \pm standard deviation (shaded intervals). See Figure S11 (SI) for examples of negative controls for 6G Quad-Stars. (c) Area fraction profiles extracted from epifluorescence microscopy time lapses depict the self-assembly kinetics of hydrogel aggregates. See control experiments in Figure S12 (SI). Unprocessed videos (bright-field and epifluorescence) are provided in the SI (Videos S1, S2, S3, and S4). (d) Schematic representation of the 3D percolating network leading to hydrogel aggregate formation in cholesterolized Quad-Stars upon addition of $\mathrm{K}^{+}$ions. (e, $\mathrm{f}$ ) Epifluorescence micrographs of 5G and 6G Quad-Star aggregates, respectively, formed after $2.5 \mathrm{~h}$ from $\mathrm{KCl}$ injection and imaged via an Alexa488 fluorophore linked to dsDNA arms. Scale bars are $50 \mu \mathrm{m}$. (g) Reversibility of assembly probed via NMM fluorimetry. Addition of $\mathrm{K}^{+}$ions (black vertical line) leads to assembly, while their chelation by cryptand injection (red vertical line) leads to fast disassembly. Depicted sample is 6G. (h) Three consecutive assembly/disassembly cycles of 6G Quad-Stars demonstrate the reversibility of hydrogel aggregate formation. See Figure S13 (SI) for zoomed-in detail of disassembly profiles. Cropped micrographs in insets (I-VI) depict sample status at assembly stages indicated by arrows. Scale bar is $50 \mu \mathrm{m}$. All insets show fields of view of the same size. Data in panels $\mathrm{c}$, g, and h are shown as mean (solid lines) \pm standard deviation (shaded regions) of at least three repeats. Area fraction profiles in panels $\mathrm{c}$ and $\mathrm{h}$ were obtained via image segmentation in FIJI (see Methods, SI). Micrographs in panels and insets were background corrected via rolling ball algorithm in FIJI (radius = 100 pixels). See Figure S14 (SI) for raw micrographs of panels e and $f$.

this framework, longer G-runs afford increased stability to transient dimers and trimers hypothesized as intermediates for G4 assembly, ${ }^{55,56}$ hence boosting folding kinetics.

In Figure $2 \mathrm{~b}(\mathrm{i})$ we explore the dependency of $t_{1 / 2}$ on oligonucleotide concentration and, expectedly, we observe a decreasing power-law trend that allows us to extract the reaction order of the assembly process as $n=3.605 \pm 0.740$ for $5 \mathrm{G}$ and $n=3.711 \pm 0.127$ for $6 \mathrm{G}$, closely matching the theoretical stoichiometry of 4 .

Figure $2 \mathrm{~b}$ (ii) proves that Quad-Stars fold significantly faster in the presence of higher potassium concentrations, with $t_{1 / 2}$ decreasing from $\sim 10 \mathrm{~h}$ to $\sim 5 \mathrm{~min}$ when $\left[\mathrm{K}^{+}\right]$is increased from 30 to $300 \mathrm{mM}$ for $6 \mathrm{G}$. A similarly strong dependence was found for $5 \mathrm{G}$.

Overall, the behaviors displayed in Figure $2 \mathrm{~b}$ demonstrate the ability of Quad-Stars to isothermally assemble in a cationand concentration-dependent fashion and, importantly, that assembly rates dramatically depend on both potassium and oligonucleotide concentrations.

We thus proceeded to investigate the ability of cholesterolized Quad-Stars to form 3D networks driven by hydrophobic 
a

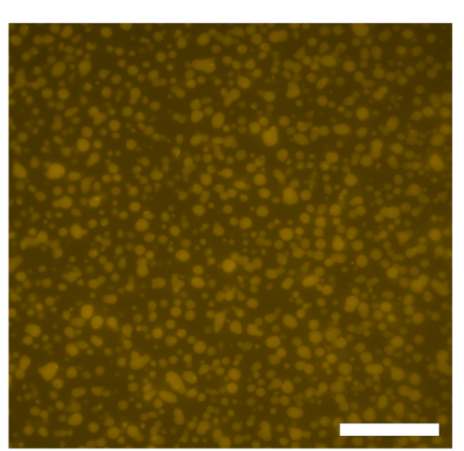

b

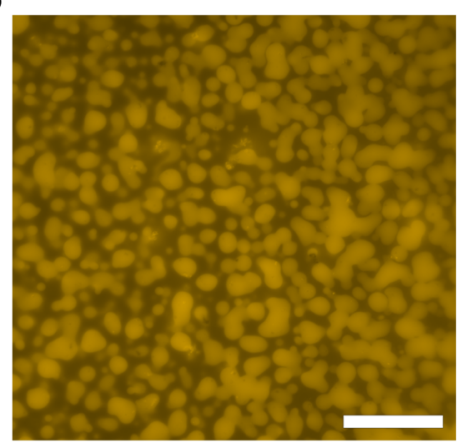

c

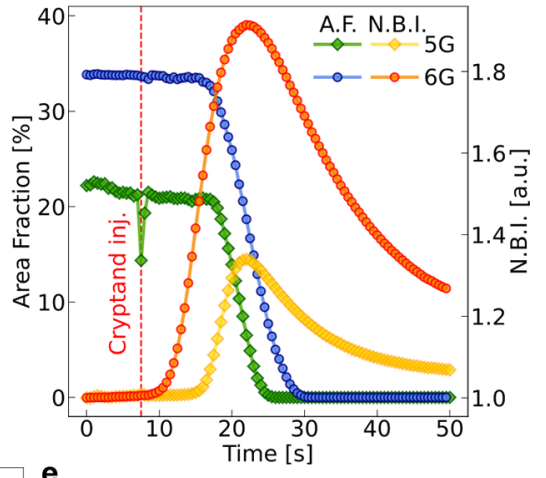

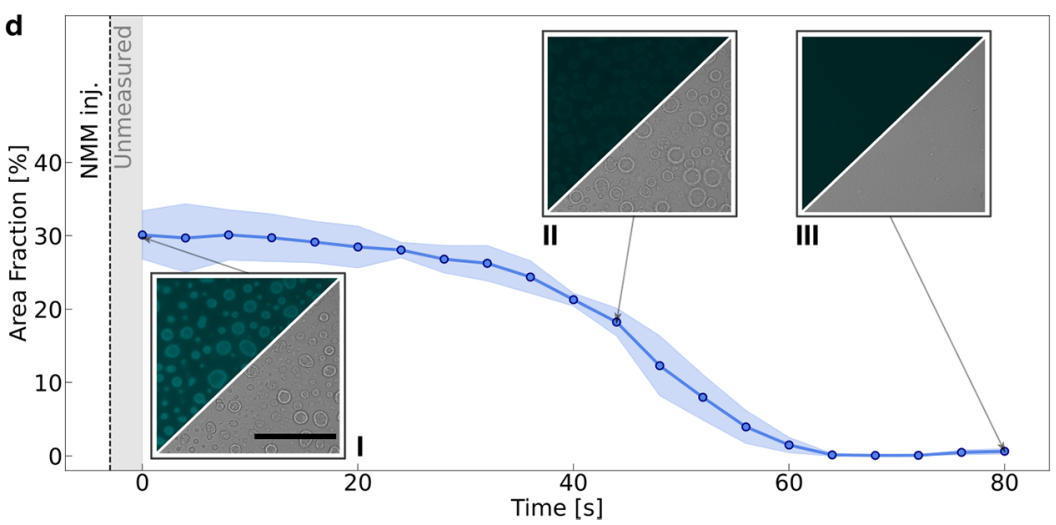

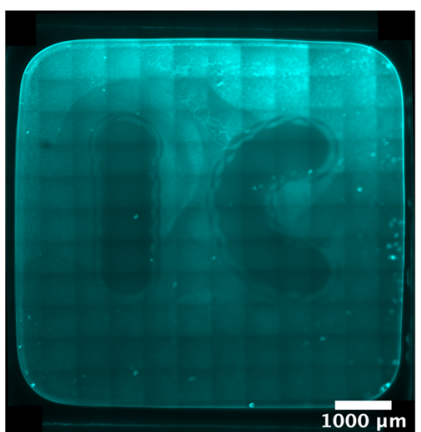

Figure 4. Uptake and release of model hydrophobic cargo rhodamine B. (a, b) Epifluorescence micrographs of 5G and 6G Quad-Star aggregates, respectively, at the end of a $3.5 \mathrm{~h}$ assembly run in the presence of rhodamine B. Hydrogel aggregates appear brighter than the background, proving preferential segregation of rhodamine B within the hydrophobic cholesterol pockets. (c) Representative profiles illustrating aggregate disassembly (A.F. $=$ area fraction) upon cryptand injection (red vertical line), thereby causing the release of the rhodamine B cargo (N.B.I. = normalized background intensity). Profiles are extracted from single FOVs of representative samples, as release timescales strongly depend on the proximity of the injection to the FOV (see Figure S15, SI). (d) Light-induced disassembly via NMM photocleavage. NMM excitation (395 nm) leads to lightinduced damage to G4 motifs and disassembly of hydrogel aggregates. Insets are composite images (bright-field and NMM fluorescence) corresponding to time steps indicated by arrows. Data are presented as mean (circles) \pm standard deviation (shaded interval) of three FOVs within the same sample (6G Quad-Stars). (e) Photoinduced engraving in a sample well, reading "IC" (Imperial College), obtained by automatically exposing the pattern to $395 \mathrm{~nm}$ LED, thereby triggering location-selective NMM-mediated photo-disassembly. Note that the underlying squarelattice pattern is an artifact of slightly uneven illumination and FOV stitching, rather than representing a physical characteristic of the sample. Scale bars are $50 \mu \mathrm{m}$, unless otherwise specified. Epifluorescence micrographs are unprocessed. Bright-field insets have been contrast-enhanced for ease of visualization. See Figure S16a,b (SI) for the raw images.

interactions, as previously observed for C-Stars ${ }^{32-34}$ and sketched in Figure $3 \mathrm{a}-\mathrm{d}$.

As for C-Stars, we expected the duplex arms to form cholesterol-induced micelles even prior to Quad-Star formation. $^{32}$ DLS in Figure S9 (SI) indeed confirms micelle formation in both $300 \mathrm{mM} \mathrm{LiCl}$ and $300 \mathrm{mM} \mathrm{KCl}$, while no micelles are observed in $2 \mathrm{mM} \mathrm{MgCl}$, probably owing to the much reduced ionic strength. Consistently, while we observe no evidence of G4 formation in noncholesterolized Quad-Stars in $\mathrm{Li}^{+}$-rich buffers, cholesterolized designs showed a moderate tendency to aggregation in $300 \mathrm{mM} \mathrm{LiCl}$, arguably due to colocalization of the G4-forming domains within micelles. In contrast, cholesterolized arms annealed in $2 \mathrm{mM} \mathrm{MgCl}_{2}$ show no aggregation and were thus chosen as the starting condition for $\mathrm{K}^{+}$-triggered network-assembly experiments.

Upon addition of $\mathrm{K}^{+}$, Quad-Star networks are expected to assemble due to G4-mediated formation of nanostar motifs, which bridge neighboring micelles. As confirmed by timeresolved NMM fluorimetry in Figure $3 b$, indeed, the addition of $300 \mathrm{mM} \mathrm{KCl}$ rapidly triggers quadruplex formation. Cholesterolized designs display substantially faster assembly compared to noncholesterolized samples, as summarized in Figure S11c (SI). The speed up is particularly noticeable for
5G samples, showing a 100-fold decrease in half-life (from $15 \mathrm{~h}$ to $10 \mathrm{~min})$. We propose that this rate increase is due to colocalization of G4-forming strands within the micelles.

NMM fluorimetry assays in Figure $3 \mathrm{~b}$ capture the isothermal folding of G4 motifs within cholesterolized Quad-Stars, rather than their aggregation into extended network phases. To verify the occurrence of the latter, and study its kinetics, isothermal assembly was monitored via epifluorescence microscopy. For these experiments, visualization was enabled by a covalently linked Alexa488 fluorophore to prevent NMM-induced photocleavage upon strong illumination (see Methods, SI).

Assembly kinetics were monitored by determining the timedependent area fraction of the microscopy field of view (FOV) occupied by aggregates (see Methods, SI). Figure 3c demonstrates a faster assembly rate for $6 \mathrm{G}$ compared to $5 \mathrm{G}$, consistent with the trends in G4-folding determined by fluorimetry (Figure $3 \mathrm{~b}$ ). Micrographs in Figure $3 \mathrm{e}, \mathrm{f}$ feature round fluorescent aggregates after $\mathrm{KCl}$ injection for both $5 \mathrm{G}$ and $6 \mathrm{G}$, with the latter displaying larger and more closely packed aggregates.

To disentangle the effect of increased ionic strength following $\mathrm{KCl}$ injection from that of G4 stabilization, in Figure S12 (SI), we monitored the response of samples 
annealed in $2 \mathrm{mM} \mathrm{MgCl}_{2}$ to the addition of $300 \mathrm{mM} \mathrm{LiCl}$. A degree of aggregation was observed, particularly with $6 \mathrm{G}$ designs, but this was significantly less pronounced than the response observed with $\mathrm{KCl}$, consistently with greater quadruplex-stabilization ability of $\mathrm{K}^{+}$and the evidence that $\mathrm{Li}^{+}$only leads to limited aggregation at equilibrium (Figure S10, SI). Consistent trends are observed in samples preannealed in $300 \mathrm{mM} \mathrm{LiCl}$ upon addition of further 300 $\mathrm{mM} \mathrm{LiCl}$ or $300 \mathrm{mM} \mathrm{KCl}$ (Figure S12, SI).

Potassium-stabilized G4 motifs are known to structurally weaken $^{57}$ and rapidly unfold ${ }^{58-62}$ upon $\mathrm{K}^{+}$removal, which should lead to Quad-Star and network disassembly. We assessed this reversibility route via NMM fluorimetry by injecting the chelating agent [2.2.2] cryptand $^{58,62}$ after a potassium-induced assembly transient, while recording NMM fluorescent emission. Figure $3 \mathrm{~g}$ shows that the disassembly is almost instantaneous upon cryptand addition, as reflected by the sudden drop in fluorescence, confirming the reversibility of Quad-Star assembly.

To further explore reversibility in Quad-Star network formation, we performed three subsequent rounds of isothermal assembly/disassembly by alternating $\mathrm{KCl}$ and cryptand injections into the same $6 \mathrm{G}$ sample. Figure $3 \mathrm{~h}$ displays the associated time-dependent area-fraction profile that demonstrates formation of visible aggregates after each $\mathrm{KCl}$ addition and an abrupt disassembly at each $\mathrm{K}^{+}$chelation. A slower assembly rate is observed for each subsequent $\mathrm{KCl}$ injection, likely due to the progressive dilution of the samples following multiple solution injections (see Methods, SI).

Therefore, we have demonstrated that Quad-Stars assemble into discrete hydrogel aggregates, with sizes ranging from a few to a few tens of micrometers, following an enzyme-free isothermal reaction. Aggregate formation is reversible and responds to changes in cation concentration, exemplified by addition and chelation of the $\mathrm{K}^{+}$ions stabilizing the inner $\mathrm{G} 4$ motifs.

The amphiphilic nature of Quad-Stars, combined with their reversibility and responsiveness, makes them promising candidates as delivery vectors for hydrophobic therapeutic molecules, which present a unique challenge in drug delivery due to their low solubility and poor bioavailability. ${ }^{63-66}$ Here, we demonstrate the ability of Quad-Stars to encapsulate and release rhodamine $\mathrm{B}$, a model hydrophobic molecule which can be easily visualized via fluorescence microscopy.

Rhodamine $\mathrm{B}$ was added to samples prepared as for the isothermal assembly experiments, prior to $\mathrm{KCl}$ injection. Microscopy images in Figure 4a,b confirm that Quad-Star aggregates are able to encapsulate hydrophobic rhodamine $B$, as demonstrated by the higher fluorescence intensity detected within the aggregates as compared to the background: $27.2 \pm$ $4.7 \%$ higher for $5 \mathrm{G}$ and $34.7 \pm 3.4 \%$ higher for $6 \mathrm{G}$.

Disassembly of the cargo-loaded hydrogels through $\mathrm{K}^{+}$ chelation leads to release of rhodamine B into solution. As demonstrated in Figure 4c, we followed the kinetics of QuadStar network disassembly and cargo release simultaneously by monitoring the aggregate area fraction and background fluorescence intensity, respectively. Here, the cryptand solution was injected in the sample well far from the imaged FOV, hence the time delay between injection and disassembly, which is then observed as a sharp drop in the area fraction. Simultaneously, the background intensity displays a sharp increase, as rhodamine is released from the aggregates within the FOV and nearby locations in the sample, before slowly decreasing as the excess dye diffuses away from the site of release.

To substantiate the applicability of Quad-Stars as drug delivery carriers, their stability in model physiological conditions was tested. Aggregates were preassembled in $\mathrm{K}^{+}$rich buffer as previously described, before undergoing supernatant removal and exchange with either phosphate buffered saline (PBS) $1 \times$, which better reflects typical cellular ionic conditions, ${ }^{67}$ or cell culture medium (Dulbecco's modified Eagle medium, DMEM) supplied with $10 \%$ or $30 \%$ fetal bovine serum (FBS), as previously done to probe the biostability of DNA nanostructures. ${ }^{6-70}$ Samples were monitored over the course of $60 \mathrm{~h}$ at room temperature. Undisturbed Quad-Star aggregates in $\mathrm{K}^{+}$-rich buffers kept growing and coarsening into a layer nearly covering the bottom of the well (Figure S17a,b, Videos S9 and S10 for 5G/6G, respectively, in the SI). Aggregates in PBS $1 \times$ neither did visibly grow nor disassemble, but coarsened and coalesced into larger microgels, indicative of an enhanced fluidity (Figure S17c,d, Videos S11 and S12 for 5G/6G, respectively, in the SI). Finally, hydrogel particles in DMEM $+10 \%$ or $30 \% \mathrm{FBS}$ fully retained their initial morphology (Figure S18a,b, Videos S13 and S14 for FBS $10 \%$ and Figure S18c,d, Videos S15 and S16 for FBS $30 \%$, for $5 \mathrm{G} / 6 \mathrm{G}$, respectively, in the SI). These results confirm the stability of Quad-Star aggregates in model physiological conditions, and thus their potential as drug delivery carriers.

Besides enabling reversible control over aggregate formation depending on ionic conditions, the bridging G4 element of Quad-Stars makes it possible to trigger disassembly through a different, orthogonal stimulus, namely UV irradiation, in the presence of NMM. While typically used as a fluorescent G4 probe (as in Figures 2 and $3 \mathrm{~b}$ ), NMM has recently been reported to induce G4-photocleavage upon strong UV exposure. $^{40}$

To test this response, we exposed assembled 6G Quad-Star hydrogels in the presence of NMM to long-wave UV (395 $\mathrm{nm}$ ), and then monitored the aggregates over time in both fluorescence (NMM emission at $670 \mathrm{~nm}$ ) and bright-field.

As shown in Figure 4d, we observe rapid disassembly of Quad-Star aggregates following injection of NMM under UV exposure, with complete dissolution occurring within $90 \mathrm{~s}$. Aggregate disassembly is observed in both fluorescence and bright-field (see Figure 4d, insets), proving that the observed effect is not due to NMM photobleaching, as also confirmed by fluorescence imaging of Alexa488-labeled samples (Figure S16c, SI). Disassembly is found to be irreversible, in agreement with literature reporting that NMM induces selective photocleavage at G4 sites following guanine photo-oxidation. ${ }^{40-44}$

A key advantage of light-induced disassembly is the possibility of activating a spatially localized response. To demonstrate this feature, we used the motorized microscope stage to expose a portion of a large 6G sample shaped as the letters "IC". Figure 4e shows selective disassembly of the Quad-Star aggregates in the exposed region and the emergence of the sought pattern.

In summary, Quad-Stars combine the robustness and versatility of amphiphilic DNA nanostars with the stimuliresponsiveness of noncanonical G4 motifs, thus allowing for controlled assembly/disassembly into hydrogel aggregates in response to biologically relevant $\mathrm{K}^{+}$cations.

We can tune the folding rates of individual motifs as well as the overall rate at which Quad-Stars aggregate into hydrogel 
particles via multiple parameters, namely potassium and oligonucleotide concentration, and the number of stacked Gquartets in the bridging G4s. The formed phases are stable under model physiological conditions and can naturally load small hydrophobic molecules, releasing them upon changes in the ionic environment. Therefore, we believe this material could be useful as the basis of drug delivery systems, exploiting cation concentration gradients existing among intra- and extracellular compartments. Moreover, in combination with a photosensitizing porphyrin ligand, Quad-Star phases can be made to irreversibly disassemble upon localized UV exposure, unlocking a further control mechanism for cargo release.

Besides the direct applicability of these responsive nanostructured phases to encapsulation and controlled release of biomedically relevant cargoes, Quad-Star aggregates may find application as scaffolds for artificial, cell-like agents that many seek to construct in the context of bottom-up synthetic biology. ${ }^{71-74}$ Here, the responsive hydrogels may act as membrane-less compartments, creating microenvironments which host active components whose action could couple with the responsiveness of the networks, hence achieving a unique synergy between structure and functionality. ${ }^{75-77}$

To this end, and to further augment the range of factors regulating Quad-Star assembly/disassembly, we envisage the possibility of exploiting helicase enzymes, either present in solution or tethered in the vicinity of the quadruplex-forming sites, or even potentially produced via cell-free protein expression systems, to selectively unfold G4s. ${ }^{78-81}$ The range of available responses could be further expanded thanks to the vast array of synthetic chemical species developed to tag, stabilize, or destabilize G-quadruplexes for molecular biology assays or therapeutics. ${ }^{82}$

Finally, we note that while this contribution focused on the assembly/disassembly thermodynamics and kinetics of QuadStar phases, the nanostructural properties of these materials remain to be discovered, particularly with respect to the ability of "regular" cholesterolized nanostars to form crystalline phases depending on design parameters such as flexibility and size. $^{33,34}$ Quad-Stars could display a similar propensity toward the formation of ordered phases, but the added complexity of the geometrical and steric constraints imposed by the G4 motif could lead to the emergence of novel crystal geometries.

\section{ASSOCIATED CONTENT}

\section{(s) Supporting Information}

The Supporting Information is available free of charge at https://pubs.acs.org/doi/10.1021/acs.nanolett.1c03314.

Experimental and data analysis methods; AGE and DLS of soluble Quad-Stars vs ionic conditions; AGE, DLS, $\mathrm{CD}$, and NMM fluorimetry vs poly-T spacer length; DLS for cholesterolized Quad-Stars; negative controls for NMM fluorimetry and self-assembly microscopy experiments; enlarged cryptand disassembly profiles; raw epifluorescence micrographs; further rhodamine B release profiles; biostability monitoring of aggregates; oligonucleotide sequences (PDF)

Video S1 showing self-assembly time lapse (bright-field) of 5G (MP4)

Video S2 showing self-assembly time lapse (Alexa488 epifluorescence) of 5G (MP4)

Video S3 showing self-assembly time lapse (bright-field) of $6 \mathrm{G}$ (MP4)
Video S4 showing self-assembly time lapse (Alexa488 epifluorescence) of 6G (MP4)

Video S5 showing rhodamine B release time lapse for 5G (MP4)

Video S6 showing rhodamine B release time lapse for 6G (MP4)

Video S7 showing bright-field time lapse of UV-triggered disassembly for 6G (MP4)

Video S8 showing NMM epifluorescence time lapse of UV-triggered disassembly for 6G (MP4)

Video S9 showing 5G after assembly and left undisturbed (negative control) (MP4)

Video S10 showing 6G after assembly and left undisturbed (negative control) (MP4)

Video S11 showing 5G after assembly and with partial replacement of supernatant with PBS (MP4)

Video S12 showing 6G after assembly and with partial replacement of supernatant with PBS (MP4)

Video S13 showing 5G after assembly and with partial replacement of supernatant with DMEM with $10 \%$ FBS (MP4)

Video S14 showing 6G after assembly and with partial replacement of supernatant with DMEM with $10 \%$ FBS (MP4)

Video S15 showing 5G after assembly and with partial replacement of supernatant with DMEM with $30 \%$ FBS (MP4)

Video S16 showing 6G after assembly and with partial replacement of supernatant with DMEM with $30 \%$ FBS (MP4)

\section{AUTHOR INFORMATION}

\section{Corresponding Authors}

Marco Di Antonio - Department of Chemistry, Imperial College London, London W12 0BZ, United Kingdom; (1) orcid.org/0000-0002-7321-1867; Email: m.di-antonio@ imperial.ac.uk

Lorenzo Di Michele - Department of Chemistry, Imperial College London, London W12 OBZ, United Kingdom; Department of Physics-Cavendish Laboratory, University of Cambridge, Cambridge CB3 OHE, United Kingdom; (1) orcid.org/0000-0002-1458-9747; Email: 1.di-michele@ imperial.ac.uk

\section{Authors}

Giacomo Fabrini - Department of Chemistry, Imperial College London, London W12 0BZ, United Kingdom

Aisling Minard - Department of Chemistry, Imperial College London, London W12 OBZ, United Kingdom

Ryan A. Brady - Department of Chemistry, King's College London, London SE1 1DB, United Kingdom; (1) orcid.org/ 0000-0002-0408-3224

Complete contact information is available at: https://pubs.acs.org/10.1021/acs.nanolett.1c03314

\section{Notes}

The authors declare no competing financial interest. Data underlying this publication are available at DOI: 10. 14469/hpc/9977 (CC-BY-NC-ND-4.0). 


\section{ACKNOWLEDGMENTS}

L.D.M. acknowledges funding from a Royal Society University Research Fellowship (UF160152) and from the European Research Council (ERC) under the Horizon 2020 Research and Innovation Programme (ERC-STG No 851667 NANOCELL). G.F. acknowledges funding from the Department of Chemistry at Imperial College London. M.D.A. and A.M. were supported by a BBSRC David Phillips Fellowship (BB/ R011605/1).

\section{REFERENCES}

(1) Zhang, F.; Nangreave, J.; Liu, Y.; Yan, H. Structural DNA Nanotechnology: State of the Art and Future Perspective. J. Am. Chem. Soc. 2014, 136, 11198-11211.

(2) Nummelin, S.; Kommeri, J.; Kostiainen, M. A.; Linko, V. Evolution of Structural DNA Nanotechnology. Adv. Mater. 2018, 30, 1703721 .

(3) Ramezani, H.; Dietz, H. Building machines with DNA molecules. Nat. Rev. Genet. 2020, 21, 5-26.

(4) Schmidt, T. L.; Beliveau, B. J.; Uca, Y. O.; Theilmann, M.; Da Cruz, F.; Wu, C. T.; Shih, W. M. Scalable amplification of strand subsets from chip-synthesized oligonucleotide libraries. Nat. Commun. 2015, 6, 8634.

(5) Marchi, A. N.; Saaem, I.; Tian, J.; Labean, T. H. One-pot assembly of a hetero-dimeric DNA origami from chip-derived staples and double-stranded scaffold. ACS Nano 2013, 7, 903-910.

(6) Liu, J. Oligonucleotide-functionalized hydrogels as stimuli responsive materials and biosensors. Soft Matter 2011, 7, 6757-6767.

(7) Xu, N.; Ma, N.; Yang, X.; Ling, G.; Yu, J.; Zhang, P. Preparation of intelligent DNA hydrogel and its applications in biosensing. Eur. Polym. J. 2020, 137, 109951.

(8) Sood, N.; Bhardwaj, A.; Mehta, S.; Mehta, A. Drug Delivery Stimuli-responsive hydrogels in drug delivery and tissue engineering Stimuli-responsive hydrogels in drug delivery and tissue engineering. Drug Delivery 2016, 23, 748-770.

(9) Nishida, Y.; Ohtsuki, S.; Araie, Y.; Umeki, Y.; Endo, M.; Emura, T.; Hidaka, K.; Sugiyama, H.; Takahashi, Y.; Takakura, Y.; Nishikawa, M. Self-assembling DNA hydrogel-based delivery of immunoinhibitory nucleic acids to immune cells. Nanomedicine 2016, 12, 123-130.

(10) Gačanin, J.; Synatschke, C. V.; Weil, T. Biomedical Applications of DNA-Based Hydrogels. Adv. Funct. Mater. 2020, 30, 1906253.

(11) Cui, J.; Wu, D.; Sun, Q.; Yang, X.; Wang, D.; Zhuang, M.; Zhang, Y.; Gan, M.; Luo, D. A PEGDA/DNA Hybrid Hydrogel for Cell-Free Protein Synthesis. Front. Chem. 2020, 8, 28.

(12) Samanta, A.; Sabatino, V.; Ward, T. R.; Walther, A. Functional and morphological adaptation in DNA protocells via signal processing prompted by artificial metalloenzymes. Nat. Nanotechnol. 2020, 15, 914-921.

(13) Lin, D. C.; Yurke, B.; Langrana, N. A. Mechanical Properties of a Reversible, DNA-Crosslinked Polyacrylamide Hydrogel. J. Biomech. Eng. 2004, 126, 104-110.

(14) Zhu, Z.; Wu, C.; Liu, H.; Zou, Y.; Zhang, X.; Kang, H.; Yang, C. J.; Tan, W. An aptamer cross-linked hydrogel as a colorimetric platform for visual detection. Angew. Chem., Int. Ed. 2010, 49, 10521056.

(15) Lin, H.; Zou, Y.; Huang, Y.; Chen, J.; Zhang, W. Y.; Zhuang, Z.; Jenkins, G.; Yang, C. J. DNAzyme crosslinked hydrogel: a new platform for visual detection of metal ions w. Chem. Commun. 2011, 47, 9312-9314.

(16) Cangialosi, A.; Yoon, C. K.; Liu, J.; Huang, Q.; Guo, J.; Nguyen, T. D.; Gracias, D. H.; Schulman, R. DNA sequence-directed shape change of photopatterned hydrogels via high-degree swelling. Science 2017, 357, 1126-1130.

(17) Tanaka, S.; Yukami, S.; Fukushima, K.; Wakabayashi, K.; Ohya, Y.; Kuzuya, A. Bulk pH-Responsive DNA Quadruplex Hydrogels
Prepared by Liquid- Phase, Large-Scale DNA Synthesis. ACS Macro Lett. 2018, 7, 295-299.

(18) Lu, C.-H.; Qi, X.-J.; Orbach, R.; Yang, H.-H.; Mironi-Harpaz, I.; Seliktar, D.; Willner, I. Switchable Catalytic Acrylamide Hydrogels Cross-Linked by Hemin/ G-Quadruplexes. Nano Lett. 2013, 13, $1298-1302$.

(19) Kahn, J. S.; Trifonov, A.; Cecconello, A.; Guo, W.; Fan, C.; Willner, I. Integration of Switchable DNA-Based Hydrogels with Surfaces by the Hybridization Chain Reaction. Nano Lett. 2015, 15, $7773-7778$.

(20) Tanaka, S.; Wakabayashi, K.; Fukushima, K.; Yukami, S.; Maezawa, R.; Takeda, Y.; Tatsumi, K.; Ohya, Y.; Kuzuya, A. Intelligent, Biodegradable, and Self-Healing Hydrogels Utilizing DNA Quadruplexes. Chem. - Asian J. 2017, 12, 2388-2392.

(21) Guo, W.; Qi, X.-J.; Orbach, R.; Lu, C.-H.; Freage, L.; MironiHarpaz, I.; Seliktar, D.; Yang, H.-H.; Willner, I. Reversible Ag + crosslinked DNA hydrogels. Chem. Commun. 2014, 50, 4065.

(22) Exon, J. H. A Review of the Toxicology of Acrylamide. J. Toxicol. Environ. Health, Part B 2006, 9, 397-412.

(23) Wang, D.; Hu, Y.; Liu, P.; Luo, D. Bioresponsive DNA Hydrogels: Beyond the Conventional Stimuli Responsiveness. Acc. Chem. Res. 2017, 50, 733-739.

(24) Um, S. H.; Lee, J. B.; Park, N.; Kwon, S. Y.; Umbach, C. C.; Luo, D. Enzyme-catalysed assembly of DNA hydrogel. Nat. Mater. 2006, 5, 797-801.

(25) Park, N.; Um, S. H.; Funabashi, H.; Xu, J.; Luo, D. A cell-free protein-producing gel. Nat. Mater. 2009, 8, 432-437.

(26) Park, N.; Kahn, J. S.; Rice, E. J.; Hartman, M. R.; Funabashi, H.; $\mathrm{Xu}, \mathrm{J}$.; Um, S. H.; Luo, D. High-yield cell-free protein production from P-gel. Nat. Protoc. 2009, 4, 1759-1770.

(27) Cheng, E.; Xing, Y.; Chen, P.; Yang, Y.; Sun, Y.; Zhou, D.; Xu, L.; Fan, Q.; Liu, D. A pH-Triggered, Fast-Responding DNA Hydrogel. Angew. Chem., Int. Ed. 2009, 48, 7660-7663.

(28) Lu, S.; Wang, S.; Zhao, J.; Sun, J.; Yang, X. A pH-controlled bidirectionally pure DNA hydrogel: Reversible self-assembly and fluorescence monitoring. Chem. Commun. 2018, 54, 4621-4624.

(29) Guo, X.; Li, F.; Liu, C.; Zhu, Y.; Xiao, N.; Gu, Z.; Luo, D.; Jiang, J.; Yang, D. Construction of Organelle-Like Architecture by Dynamic DNA Assembly in Living Cells. Angew. Chem., Int. Ed. 2020, 59, 20651-20658.

(30) Simon, A. J.; Walls-Smith, L. T.; Plaxco, K. W. Exploiting the conformational-selection mechanism to control the response kinetics of a "smart" DNA hydrogel. Analyst 2018, 143, 2531-2538.

(31) Oishi, M.; Nakatani, K. Dynamically Programmed Switchable DNA Hydrogels Based on a DNA Circuit Mechanism. Small 2019, $15,1900490$.

(32) Brady, R. A.; Brooks, N. J.; Cicuta, P.; Di Michele, L. Crystallization of Amphiphilic DNA C-Stars. Nano Lett. 2017, 17, 3276-3281.

(33) Brady, R. A.; Brooks, N. J.; Foderà, V.; Cicuta, P.; Di Michele, L. Amphiphilic-DNA Platform for the Design of Crystalline Frameworks with Programmable Structure and Functionality. J. Am. Chem. Soc. 2018, 140, 15384-15392.

(34) Brady, R. A.; Kaufhold, W. T.; Brooks, N. J.; Fodera, V.; Di Michele, L. Flexibility defines structure in crystals of amphiphilic DNA nanostars. J. Phys.: Condens. Matter 2019, 31, 074003.

(35) Majewski, P. W.; Michelson, A.; Cordeiro, M. A. L.; Tian, C.; Ma, C.; Kisslinger, K.; Tian, Y.; Liu, W.; Stach, E. A.; Yager, K. G.; Gang, O. Resilient three-dimensional ordered architectures assembled from nanoparticles by DNA. Sci. $A d v$. 2021, 7, eabf0617.

(36) Kahn, J. S.; Gang, O. Designer Nanomaterials through Programmable Assembly. Angew. Chem. Int. Ed. 2021, 60, 2-21.

(37) Tian, Y.; Lhermitte, J. R.; Bai, L.; Vo, T.; Xin, H. L.; Li, H.; Li, R.; Fukuto, M.; Yager, K. G.; Kahn, J. S.; Xiong, Y.; Minevich, B.; Kumar, S. K.; Gang, O. Ordered three-dimensional nanomaterials using DNA-prescribed and valence-controlled material voxels. Nat. Mater. 2020, 19, 789-796.

(38) Linko, V.; Kostiainen, M. A. De novo nanomaterial crystals from DNA frameworks. Nat. Mater. 2020, 19, 706-707. 
(39) Wang, S.-T.; Minevich, B.; Liu, J.; Zhang, H.; Nykypanchuk, D.; Byrnes, J.; Liu, W.; Bershadsky, L.; Liu, Q.; Wang, T.; Ren, G.; Gang, O. Designed and biologically active protein lattices. Nat. Commun. 2021, 12, 3702

(40) McBrayer, D.; Schoonover, M.; Long, K. J.; Escobedo, R.; Kerwin, S. M. N-Methylmesoporphyrin IX Exhibits G-QuadruplexSpecific Photocleavage Activity. ChemBioChem 2019, 20, 1924-1927.

(41) Morikawa, M.; Kino, K.; Oyoshi, T.; Suzuki, M.; Kobayashi, T.; Miyazawa, H. Product analysis of photooxidation in isolated quadruplex DNA; 8-oxo-7,8-dihydroguanine and its oxidation product at $3=\mathrm{G}$ are formed instead of 2,5-diamino- $4 \mathrm{H}$-imidazol-4-one. RSC Adv. 2013, 3, 25694-25697.

(42) Morikawa, M.; Kino, K.; Oyoshi, T.; Suzuki, M.; Kobayashi, T.; Miyazawa, H. Analysis of Guanine Oxidation Products in DoubleStranded DNA and Proposed Guanine Oxidation Pathways in SingleStranded, Double-Stranded or Quadruplex DNA. Biomolecules 2014, $4,140-159$.

(43) Kawauchi, K.; Urano, R.; Kinoshita, N.; Kuwamoto, S.; Torii, T.; Hashimoto, Y.; Taniguchi, S.; Tsuruta, M.; Miyoshi, D. Photosensitizers based on g-quadruplex ligand for cancer photodynamic therapy. Genes 2020, 11, 1340.

(44) Cadet, J.; Wagner, J. R. DNA base damage by reactive oxygen species, oxidizing agents, and UV radiation. Cold Spring Harbor Perspect. Biol. 2013, 5, a012559.

(45) Burge, S.; Parkinson, G. N.; Hazel, P.; Todd, A. K.; Neidle, S. Quadruplex DNA: Sequence, topology and structure. Nucleic Acids Res. 2006, 34, 5402-5415.

(46) Mergny, J. L.; De Cian, A.; Ghelab, A.; Saccà, B.; Lacroix, L. Kinetics of tetramolecular quadruplexes. Nucleic Acids Res. 2005, 33, 81-94.

(47) Bhattacharyya, D.; Mirihana Arachchilage, G.; Basu, S. Metal cations in G-quadruplex folding and stability. Front. Chem. 2016, 4, 38.

(48) Li, Y.; Geyer, C. R.; Sen, D. Recognition of Anionic Porphyrins by DNA Aptamers. Biochemistry 1996, 35, 6911-6922.

(49) Ren, J.; Chaires, J. B. Sequence and structural selectivity of nucleic acid binding ligands. Biochemistry 1999, 38, 16067-16075.

(50) Nicoludis, J. M.; Miller, S. T.; Jeffrey, P. D.; Barrett, S. P.; Rablen, P. R.; Lawton, T. J.; Yatsunyk, L. A. Optimized End-Stacking Provides Specificity of N-Methyl Mesoporphyrin IX for Human Telomeric G-Quadruplex DNA. J. Am. Chem. Soc. 2012, 134, 2044620456.

(51) Bardin, C.; Leroy, J. L. The formation pathway of tetramolecular G-quadruplexes. Nucleic Acids Res. 2008, 36, 477-488.

(52) Mergny, J. L.; Sen, D. DNA quadruple helices in nanotechnology. Chem. Rev. 2019, 119, 6290-6325.

(53) Pörschke, D.; Eigen, M. Co-operative non-enzymatic base recognition III. Kinetics of the helix-coil transition of the oligoribouridylic - oligoriboadenylic acid system and of oligoriboadenylic acid alone at acidic pH. J. Mol. Biol. 1971, 62, 361-381.

(54) Wyatt, J. R.; Davis, P. W.; Freier, S. M. Kinetics of G-QuartetMediated Tetramer Formation. Biochemistry 1996, 35, 8002-8008.

(55) Mondal, S.; Bhat, J.; Jana, J.; Mukherjee, M.; Chatterjee, S. Reverse Watson-Crick $G-G$ base pair in G-quadruplex formation. Mol. BioSyst. 2016, 12, 18-22.

(56) Štefl, R. S.; Cheatham, T. E., III; Špačková, S.; Fadrná, E.; Berger, I.; Koča, J.; Šponer, J. Formation Pathways of a GuanineQuadruplex DNA Revealed by Molecular Dynamics and Thermodynamic Analysis of the Substates. Biophys. J. 2003, 85, 1787-1804.

(57) Bergues-Pupo, A. E.; Arias-Gonzalez, J. R.; Morón, M. C.; Fiasconaro, A.; Falo, F. Role of the central cations in the mechanical unfolding of DNA and RNA G-quadruplexes. Nucleic Acids Res. 2015, $43,7638-7647$.

(58) Aleman-Garcia, M. A.; Orbach, R.; Willner, I. Ion-responsive hemin-G-quadruplexes for switchable DNAzyme and enzyme functions. Chem. - Eur. J. 2014, 20, 5619-5624.

(59) Aizen, R.; Golub, E.; Trifonov, A.; Shimron, S.; Niazov-Elkan, A.; Willner, I. G-Quadruplex-Stimulated Optical and Electrocatalytic DNA Switches. Small 2015, 11, 3654-3658.
(60) Olejko, L.; Cywiński, P. J.; Bald, I. An ion-controlled four-color fluorescent telomeric switch on DNA origami structures. Nanoscale 2016, 8, 10339-10347.

(61) Liu, X.; Zhang, J.; Fadeev, M.; Li, Z.; Wulf, V.; Tian, H.; Willner, I. Chemical and photochemical DNA "gears" reversibly control stiffness, shape-memory, self-healing and controlled release properties of polyacrylamide hydrogels $\dagger$. Chem. Sci. 2019, 10, 10081016

(62) Chan, M. S.; Leung, H. M.; Wong, S. W.; Lin, Z.; Gao, Q.; Chang, T. J. H.; Lai, K. W. C.; Lo, P. K. Reversible reconfiguration of high-order DNA nanostructures by employing G-quartet toeholds as adhesive units. Nanoscale 2020, 12, 2464-2471.

(63) Gulyakin, I. D.; Oborotova, N. A.; Pechennikov, V. M. Solubilization of hydrophobic antitumor drugs (review). Pharm. Chem. J. 2014, 48, 209-213.

(64) Sareen, S.; Joseph, L.; Mathew, G. Improvement in solubility of poor water-soluble drugs by solid dispersion. Int. J. Pharm. Investig. 2012, 2, 12 .

(65) Savjani, K. T.; Gajjar, A. K.; Savjani, J. K. Drug Solubility: Importance and Enhancement Techniques. ISRN Pharm. 2012, 2012, $1-10$.

(66) Kalepu, S.; Nekkanti, V. Insoluble drug delivery strategies: Review of recent advances and business prospects. Acta Pharm. Sin. B 2015, 5, 442-453.

(67) Thier, S. O. Potassium physiology. Am. J. Med. 1986, 80, 3-7. (68) Chandrasekaran, A. R. Nuclease resistance of DNA nanostructures. Nat. Rev. Chem. 2021, 5, 225-239.

(69) Hahn, J.; Wickham, S. F. J.; Shih, W. M.; Perrault, S. D. Addressing the Instability of DNA Nanostructures in Tissue Culture. ACS Nano 2014, 8, 8765-8775.

(70) Wang, S.-T.; Gray, M. A.; Xuan, S.; Lin, Y.; Byrnes, J.; Nguyen, A. I.; Todorova, N.; Stevens, M. M.; Bertozzi, C. R.; Zuckermann, R. $\mathrm{N}$.; Gang, O. DNA origami protection and molecular interfacing through engineered sequence-defined peptoids. Proc. Natl. Acad. Sci. U. S. A. 2020, 117, 6339-6348.

(71) Perrault, S. D.; Shih, W. M. Virus-inspired membrane encapsulation of DNA nanostructures to achieve in vivo stability. ACS Nano 2014, 8, 5132-5140.

(72) Zhang, Z.; Yang, Y.; Pincet, F.; Llaguno, M. C.; Lin, C. Placing and shaping liposomes with reconfigurable DNA nanocages. Nat. Chem. 2017, 9, 653-659.

(73) Kurokawa, C.; Fujiwara, K.; Morita, M.; Kawamata, I.; Kawagishi, Y.; Sakai, A.; Murayama, Y.; Nomura, S. I. M.; Murata, S.; Takinoue, M.; Yanagisawa, M. DNA cytoskeleton for stabilizing artificial cells. Proc. Natl. Acad. Sci. U. S. A. 2017, 114, 7228-7233.

(74) Göpfrich, K.; Platzman, I.; Spatz, J. P. Mastering Complexity: Towards Bottom-up Construction of Multifunctional Eukaryotic Synthetic Cells. Trends Biotechnol. 2018, 36, 938-951.

(75) Buddingh', B. C.; Van Hest, J. C. M. Artificial Cells: Synthetic Compartments with Life-like Functionality and Adaptivity. Acc. Chem. Res. 2017, 50, 769-777.

(76) Jeong, S.; Nguyen, H. T.; Kim, C. H.; Ly, M. N.; Shin, K. Toward Artificial Cells: Novel Advances in Energy Conversion and Cellular Motility. Adv. Funct. Mater. 2020, 30, 1907182.

(77) Yewdall, N. A.; André, A. A.; Lu, T.; Spruijt, E. Coacervates as models of membraneless organelles. Curr. Opin. Colloid Interface Sci. 2021, 52, 101416.

(78) Sanders, C. M. Human Pif1 helicase is a G-quadruplex DNAbinding protein with G-quadruplex DNA-unwinding activity. Biochem. J. 2010, 430, 119-128.

(79) Wu, C. G.; Spies, M. G-quadruplex recognition and remodeling by the FANCJ helicase. Nucleic Acids Res. 2016, 44, 8742-8753.

(80) Sauer, M.; Paeschke, K. G-quadruplex unwinding helicases and their function in vivo. Biochem. Soc. Trans. 2017, 45, 1173-1182.

(81) Byrd, A. K.; Bell, M. R.; Raney, K. D. Pif1 helicase unfolding of G-quadruplex DNA is highly dependent on sequence and reaction conditions. J. Biol. Chem. 2018, 293, 17792-17802. 
(82) Raguseo, F.; Chowdhury, S.; Minard, A.; Di Antonio, M. Chemical-biology approaches to probe DNA and RNA G-quadruplex structures in the genome. Chem. Commun. 2020, 56, 1317-1324.

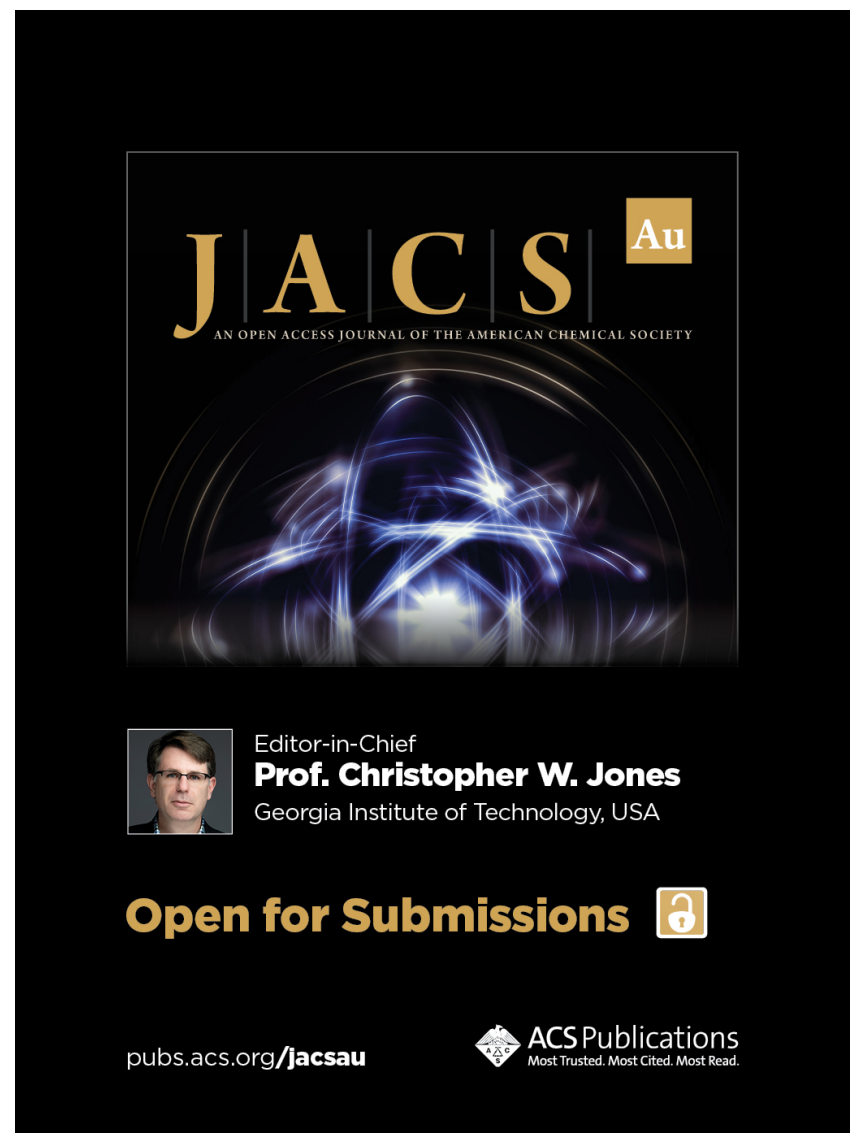

